\begin{tabular}{|c|l|}
\hline Title & Distributed Observer over Delayed Sensor Networks for Systems with Unknown Inputs \\
\hline Author(s) & A dachi, Ry osuke; Y amashita, Yuh; Kobay ashi, Koichi \\
\hline Citation & $\begin{array}{l}\text { IEICE transactions on fundamentals of electronics communications and computer sciences, E103.A (2), 469-477 } \\
\text { https://doi.org/10.1587/transfun.2019MA P0012 }\end{array}$ \\
\hline Issue Date & 2020-02 \\
\hline Doc URL & http://hdl.handle.net/2115/76922 \\
\hline Rights & copyright@2020 IEICE \\
\hline Type & article \\
\hline File Information & Distributed Observer over Delayed Sensor Networks for Systems with Unknown Inputs.pdf \\
\hline
\end{tabular}

Instructions for use 


\title{
Distributed Observer over Delayed Sensor Networks for Systems with Unknown Inputs
}

\author{
Ryosuke ADACHI $^{\dagger \text { a) }}$, Nonmember, Yuh YAMASHITA ${ }^{\dagger \dagger}$, and Koichi KOBAYASHI $^{\dagger \dagger}$, Members
}

\begin{abstract}
SUMMARY In this paper, we consider the design problem of an unknown-input observer for distributed network systems under the existence of communication delays. In the proposed method, each node estimates all states and calculates inputs from its own estimate. It is assumed that the controller of each node is given by an observer-based controller. When calculating each node, the input values of the other nodes cannot be utilized. Therefore, each node calculates alternative inputs instead of the unknown inputs of the other nodes. The alternative inputs are generated by own estimate based on the feedback controller of the other nodes given by the assumption. Each node utilizes these values instead of the unknown inputs when calculating the estimation and delay compensation. The stability of the estimation error of the proposed observer is proven by a LyapunovKrasovskii functional. The stability condition is given by a linear matrix inequality (LMI). Finally, the result of a numerical simulation is shown to verify the effectiveness of the proposed method.

key words: sensor network, data aggregation, communication delay, distributed estimation
\end{abstract}

\section{Introduction}

In recent years, sensor networks that include many sensors have attracted significant attention. Many sensors contribute to improving the sensing accuracy in a sensor network. Fault-tolerant sensing can be realized with redundant sensors. Past studies of applications to sensor networks have the most interest about communication efficiencies. To reduce communication traffic, distributed consensus algorithms have been proposed. In [1]-[4], a distributed observer that combines a consensus and Kalman filters was proposed. The consensus filter can calculate an average consensus value from the communication between neighbor nodes in a network. An estimate of the observer proposed by [1]-[4] is calculated from a Kalman filter based on the consensus value. A gossip algorithm [5] is also a distributed consensus algorithm for a sensor network. In this algorithm, each node selects received data from the neighbor nodes in the network at random. Finally, each node obtains the consensus value with less communication. When there are communication delays in the network, a data aggregation protocol is useful for collecting the information of the other nodes. We can calculate the estimate from aggregated data by using a delay-compensated observer. In addition, it was

Manuscript received April 14, 2019.

Manuscript revised October 10, 2019.

${ }^{\dagger}$ The author is with Yamaguchi University, Ube-shi, 755-8611 Japan.

${ }^{\dagger}$ The authors are with Hokkaido University, Sapporo-shi, 0600814 Japan.

a)E-mail: r-adachi@yamaguchi-u.ac.jp DOI: 10.1587/transfun.2019MAP0012 indicated in [6] that a data aggregation protocol can reduce the power consumption of each node.

Distributed estimation in sensor networks can be applied to distributed control systems. In observer-based distributed control, each estimator that is collocated with the actuator has to estimate the state without inputs from the other actuators except for its own node. For these reasons, some unknown input observers were proposed in past studies. In [7], a delayed unknown input observer for a discretetime system was proposed. The delayed unknown input observer provides only a delayed estimate. However, this observer can reconstruct the unknown inputs with delays. On the other hand, in [8], [9], unknown input observers for a continuous-time system were proposed. These observers can calculate the estimate from the no delay output without some inputs. However, the unknown input observer proposed in [8], [9] cannot calculate the state in real time under the existence of a communication delay.

In our past work, we proposed a data-aggregationbased estimation for a sensor network with a communication delay in [10]. In this work, it is assumed that each node is connected by the actuator network without a communication delay. Therefore, all nodes can obtain all input values of the other nodes and estimate the state from the delay-compensated observer. However, in many cases, the assumption of an actuator network without a communication delay is not satisfied. Thus, in this paper, we consider unknown input observer problems in a sensor network with communication delays. The communication delays depend on communication paths in the network. Therefore, we need a multi-delay-compensated observer for estimation in the network. A multi-delay-compensated observer was also proposed in [11], [12]. However, both observers utilize a memory of the inputs to compensate the delay included in the outputs. Thus, instead of an unknown input, an alternative input is needed in the calculation of unknown input observers with a delay compensation.

In this paper, we propose an unknown input observer by using alternative inputs. In our method, all nodes calculate full states from the information obtained from the sensor network. It is assumed that an input of each node is generated by an observer-based controller. Thus, each node calculates the input values from its own estimate. Instead of the inputs generated by the other nodes, each node calculates the alternative inputs from its own estimate based on the feedback controller of the other node given by the assumption. We expect that the alternative inputs converge to a real in- 
put of each node if the converge rates of each observer are sufficiently high. The estimate and delay-compensated term of each node are calculated from the alternative inputs instead of the unknown inputs. The stability of the estimation error of the proposed observer is proven by the LyapunovKrasovskii functional proposed in [13]. Finally, the stability condition of the proposed observer is given by LMIs.

The paper is organized as follows. The problem and some assumptions are explained in Sect. 2. We provide details of the alternative input observer and present the main results in Sect. 3. The results of a numerical simulation are shown in Sect. 4. Section 5 concludes the paper.

\section{Notation and Problem Setting}

\subsection{Notation}

We utilize $\mathbb{S}_{++}^{n}$ as a set of $n$-dimensional positive definite matrices. The function $\mathrm{He}(\cdot)$ means $\mathrm{He}(X)=X+X^{T}$. The notation diag $\left(A_{i}\right)_{i \in S}$ denotes a matrix arranged $A_{i}$ diagonally, where $S$ is an index set. Similarly, row $\left(A_{i}\right)_{i \in S}$ denotes a matrix arranged $A_{i}$ horizontally. For example, if $S=\{1,2,3\}$, then $\operatorname{diag}\left(A_{i}\right)_{i \in S}$ and row $\left(A_{i}\right)_{i \in S}$ are

$$
\begin{aligned}
\operatorname{diag}\left(A_{i}\right)_{i \in S} & =\left(\begin{array}{ccc}
A_{1} & 0 & 0 \\
0 & A_{2} & 0 \\
0 & 0 & A_{3}
\end{array}\right), \\
\operatorname{row}\left(A_{i}\right)_{i \in S} & =\left(\begin{array}{lll}
A_{1} & A_{2} & A_{3}
\end{array}\right) .
\end{aligned}
$$

\subsection{Problem Formulation}

We consider a distributed network system as shown in Fig. 1. The network has $N$ nodes, which include sensors and actuators. Each node collects the observed values of the other nodes via a network and estimates a state. The input of each node is calculated from the estimate of its own node, as shown in Fig. 2. The dynamics of the plant in Fig. 1 can be represented by

$$
\begin{aligned}
& \dot{x}=A x+\sum_{i=1}^{N} B_{i} u_{i} \\
& y=C x
\end{aligned}
$$

where $x \in \mathbb{R}^{n}$ is the state, and $u_{i} \in \mathbb{R}^{m_{i}}$ is the distributed input of node i. $y \in \mathbb{R}^{p}$ is the output of the system. The output $y$ consists of all redundant raw measurements. Therefore, rank $C$ may be less than $p$, and $p$ may be larger than $n$.

To represent the network, we utilize an undirected graph. The set of nodes is defined by $V:=\{1,2, \ldots, N\}$, and the set of edges is defined by $E \subseteq V \times V$. From $V$ and $E$, the network is expressed by $G:=(V, E)$. In this paper, it is assumed that $G$ is connected. If $G$ is connected, then there exists at least one tree $T:=(V, \hat{E})$ that is a subgraph of $G$, where $\hat{E}$ is a set of the edges that satisfy $\hat{E} \subseteq E$.

The nodes that have their own sensors provide measurements to the sensor network. Let us define the scalable

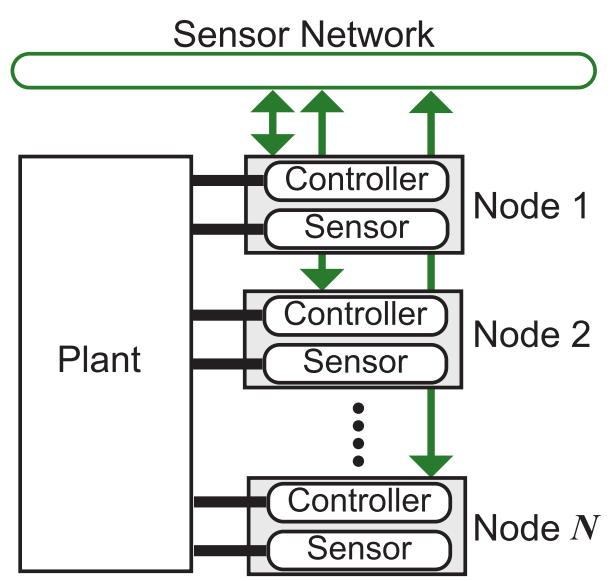

Fig. 1 Distributed network system.

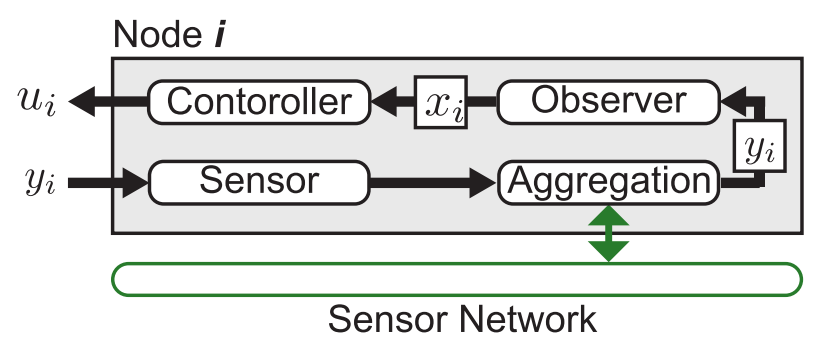

Fig. 2 Detail of each node.

communication as follows.

Definition 1 (Scalable Communication on $T$ ). When the dimension of communication data on all edges in $\hat{E}$ is independent of $N=|V|$, we call the communication scalable.

This paper considers aggregations of the sensor measurements by scalable communications. The redundant raw output $y$ is aggregated into a $q$-dimensional vector

$$
y_{\text {agr }}=F y=C_{\text {agr }} x,
$$

where $F \in \mathbb{R}^{q \times p}$ represents the relationship between $y$ and $y_{\text {agr }}$, and $C_{\text {agr }}=F C$. By renumbering the elements of $y$, we can decompose the matrix $F$ as

$$
F=\left[\begin{array}{l|l|l}
F_{1} & \cdots & F_{N}
\end{array}\right],
$$

where $F_{i}$ corresponds to the output of the $i$-th node. Therefore, the outputs of the nodes, which are mapped to the aggregated output space, are defined as

$$
\begin{aligned}
& y_{1}=\left[F_{1}|0| \cdots \mid 0\right] y=C_{1} x \\
& \vdots \\
& y_{N}=\left[0|\cdots| 0 \mid F_{N}\right] y=C_{N} x,
\end{aligned}
$$

where $C_{i}=\left[0|\cdots| 0\left|F_{i}\right| 0|\cdots| 0\right] C$. If one can obtain the current outputs $y_{i}$ with no transmission delay, then the aggregated output of $y_{i}$ coincides with $y_{\text {agr }}$, i.e., 


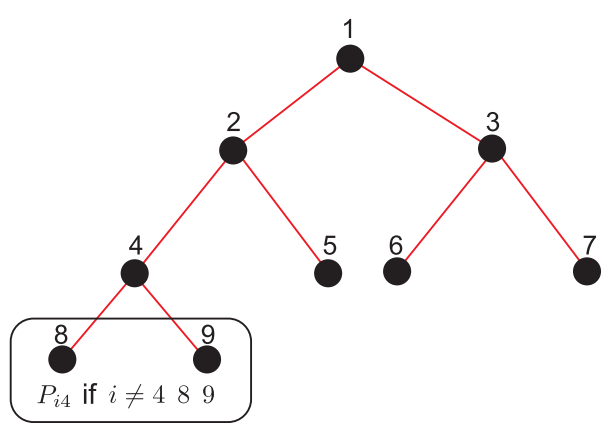

Fig. 3 Example of tree network.

$$
y_{\text {agr }}=\sum_{i=1}^{N} y_{i} .
$$

We assume that the physical meaning of measurements should be preserved in the aggregation. For example, the aggregations of positions and velocities are prohibited by this assumption. In addition, we also prohibit aggregations that cancel the measurements each other.

The nodes that have actuators calculate the input values from an estimate of the state. The estimate of node $i$ is denoted by $\hat{x}_{i}$. Based on $\hat{x}_{i}$, the input of node $i$ is given by

$$
u_{i}=K_{i} \hat{x}_{i} .
$$

The nodes that have no sensors provide no information to the sensor network, and the corresponding $F_{i}$ has no column. Therefore, we model them as nodes whose output matrices are zero matrices, i.e., $C_{i}=0$.

In the sensor network, each node communicates the information of the measurements to the other nodes via the paths in $E$. We define a set of neighbors of node $i$ connected by $T$ as $J_{i}:=\{j ;(i, j) \in \hat{E}\}$. Node $i$ can mutually communicate to the nodes included by $J_{i}$. Once a root of the undirected tree $T$ is fixed, a parent node and child nodes of each node are determined. The set of child nodes of node $j$ is represented by $P_{i j}$, where $i$ is a fixed root node. In Fig. 3 , if $i \neq 4,8,9$, then the set of child nodes in node 4 is $P_{i 4}=\{8,9\}$.

A communication delay from node $i$ to $j$ is denoted by $d_{(i, j)}$. In the case where $j \notin J_{i}, d_{(i, j)}$ represents a total delay on the simple path from node $i$ to $j$. For example, $d_{(1,8)}$ in Fig. 3 is $d_{(1,8)}=d_{(1,2)}+d_{(2,4)}+d_{(4,8)}$.

Each node collects the observed data of the other nodes through a data aggregation protocol:

$$
\begin{aligned}
& \bar{y}_{i j}(t)=y_{i}(t)+\sum_{k \in J_{i} \backslash\{j\}} \bar{y}_{k i}\left(t-d_{(k, i)}\right), \\
& \hat{y}_{i}(t)=y_{i}(t)+\sum_{j \in J_{i}} \bar{y}_{j i}\left(t-d_{(j, i)}\right),
\end{aligned}
$$

where $\bar{y}_{i j}$ is the send data from node $i$ to $j$, and $\hat{y}_{i}$ is an aggregated output in node $i$. We can represent $\hat{y}_{i}$ as

$$
\hat{y}_{i}(t)=\sum_{j=1}^{N} C_{i} x\left(t-d_{(i, j)}\right) .
$$

When the communication delay is sufficiently small to be ignored, $\hat{y}_{i}$ for all $i$ becomes $y_{\text {agr }}$.

If $\left(A, C_{i}\right)$ is an observable pair for all $i \in V$, then each node does not require communication over the network. If this assumption is not satisfied, then all nodes need to collect other measurements to estimate the states. In addition, we can obtain some benefits, for example, fault tolerance and the reduction of observation noises, from using all measurements. However, to collect all measurements individually, we require a significant amount of communication traffic. Therefore, data aggregation is important over sensor networks.

The main problem is the design of a distributed observer with data aggregation over delayed sensor networks. To design the observer gains $L_{1}, \cdots, L_{N}$, which are defined in Sect.3.2, a set of system parameters $\Lambda:=$ $\left\{A, T,\left\{d_{(i, j)} ;(i, j) \in E\right\},\left\{B_{i}, K_{i}, C_{i} ; i \in V\right\}\right\}$ is required. In the calculation of estimates, node $i$ can utilize aggregated output $\hat{y}_{i}(t)$ and histories of its own input $u_{i}(\tau)$ and estimate $\hat{x}_{i}(\tau)$, $\tau \in\left[t, t-d_{(i, \max )}\right]$, where $d_{(i, \max )}$ is the maximum delay expressed by $d_{(i, \max )}=\max _{j \in J_{i}} d_{(j, i)}$. Then, the main problem is expressed as follows:

Problem 1. For a given set of system parameters $\Lambda$, find distributed observers

$$
\begin{aligned}
\dot{\hat{x}}_{i}(t)=f_{i}\left(\hat{x}_{i}(t), u_{i}(t), \hat{y}_{i}(t), \hat{\Xi}_{i}(t)\right), \\
\hat{\Xi}_{i}(t)=\hat{h}_{i}\left(\left\{\bar{\Xi}_{j i}\left(t-d_{(j, i)}\right) ; j \in J_{i}\right\},\right. \\
\left.\left\{\hat{x}_{i}(\tau) ; \tau \in\left[t, t-d_{(i, \max )}\right]\right\}\right), \\
\bar{\Xi}_{i j}(t)=\bar{h}_{i j}\left(\left\{\bar{\Xi}_{k i}\left(t-d_{(k, i)}\right) ; j \in J_{i},\right.\right. \\
\left.\left\{\hat{x}_{i}(\tau) ; \tau \in\left[t, t-d_{(i, \text { max })}\right]\right\}\right)
\end{aligned}
$$

such that

$$
\lim _{t \rightarrow \infty}\left(x(t)-\hat{x}_{i}(t)\right)=0 .
$$

Remark 1. In many cases, it is assumed that $d_{(i, j)}=$ $d_{(j, i)}$. This is a natural assumption. However, our proposed method does not need this assumption.

\section{Alternative Input Observer for Distributed Network Systems}

\subsection{Alternative Input Observer with No Delays}

In the case that $d_{(i, j)}=0$, Problem 1 becomes 2 . Consider the following problem:

Problem 2. Solve Problem 1 under the assumption that the communication delay $d_{(i, j)}$ is 0 .

In [8], [9], Problem 2 is considered an unknown input observer problem, and a linear system with unknown inputs is expressed as

$$
\begin{aligned}
& \dot{x}=A x+B u+D v, \\
& y=C x,
\end{aligned}
$$


where $u$ is a known input, and $v$ is an unknown input. In the problem of this paper,

$$
B u=B_{i} u_{i}, D=\sum_{i \neq j} B_{j}, C=C_{\mathrm{agr}} .
$$

The unknown input observer of system (8) can be expressed by

$$
\begin{aligned}
& \dot{z}=N z+L y+G u, \\
& \hat{x}=z-E y .
\end{aligned}
$$

In [9], a stability condition of the observer (9) is indicated as algebraic equations of $N, L, G$, and $E$. On the other hand, in [8], the stability condition of the observer (9) is shown as the LMI. A difference between a general unknown input observer and our problem is an assumption that the input of each node is given by (5). Note that the estimate of each node converges to $\hat{x}_{1}=\cdots=\hat{x}_{N}=x$ if the estimation errors of each node are stable. If the convergence rates of the estimation errors are sufficiently high, then we can expect that each node can estimate the unknown input of the other nodes from its own estimate. Therefore, we utilize an alternative input $\hat{u}_{i j}$ instead of $u_{j}$ in the calculation of the observer on node $i$. By using $\hat{x}_{i}$, the alternative input $\hat{u}_{i j}$ is calculated by

$$
\hat{u}_{i j}=K_{j} \hat{x}_{i} \text {. }
$$

Based on the alternative inputs, the observer in node $i$ can be expressed by

$$
\dot{\hat{x}}_{i}=A \hat{x}_{i}+B_{i} u_{i}+\sum_{i \neq j} B_{i} \hat{u}_{i j}+L_{i}\left(\hat{y}_{i}-C_{\mathrm{agr}} \hat{x}_{i}\right) .
$$

The stability condition of the estimation error on (10) is given by Theorem 1 .

Let $\bar{B}_{i}$ be a matrix in which $B_{j} K_{j}$ are horizontally arranged except for the $i$ th block element. In the $\bar{B}_{i}$, the $i$ th block element is the negative sum of $B_{j} K_{j}$ except for $B_{i} K_{i}$. Thus, $\bar{B}_{i}$ can be expressed by

$$
\bar{B}_{i}=\left[B_{1} K_{1}, \cdots, B_{i-1} K_{i-1},-\sum_{i \neq j} B_{j} K_{j}, \cdots, B_{N} K_{N}\right] .
$$

For example, if $N=3$, then $\bar{B}_{2}$ can be expressed by

$$
\bar{B}_{2}=\left[B_{1} K_{1},-\left(B_{1} K_{1}+B_{3} K_{3}\right), B_{3} K_{3}\right] \text {. }
$$

By using $\bar{B}_{i}, \bar{A}$ is defined by

$$
\bar{A}=\operatorname{diag}(A)_{i \in V}-\left(\begin{array}{c}
\bar{B}_{1} \\
\vdots \\
\bar{B}_{N}
\end{array}\right) .
$$

Theorem 1. If there exist $P_{i} \in \mathbb{S}_{++}^{n}$ and the observer gains $L_{i}$ that satisfy

$$
\operatorname{He}\left(\operatorname{diag}\left(P_{i}\right)_{i \in V}\left(\bar{A}-\operatorname{diag}\left(L_{i} C_{\text {ide }}\right)_{i \in V}\right)\right)<0,
$$

then the estimation error $\xi_{i}=x-\hat{x}_{i}$ of the observer (10) is stable.

Proof. Let $\xi \in \mathbb{R}^{n N}$ be a vector that is expressed by

$$
\xi=\left[\xi_{1}^{T}, \cdots, \xi_{N}^{T}\right]^{T} .
$$

By using $\xi$, the error dynamics of (10) can be expressed by

$$
\dot{\xi}=\left(\bar{A}-\operatorname{diag}\left(L_{i} C_{\text {agr }}\right)_{i \in V}\right) \xi .
$$

We consider a Lyapunov function candidate $V$ as

$$
V=\xi^{T} \operatorname{diag}\left(P_{i}\right)_{i \in V} \xi .
$$

By time-differentiating $V$, we can get

$$
\dot{V}=\xi^{T} \operatorname{He}\left(\operatorname{diag}\left(P_{i}\right)_{i \in V}\left(\bar{A}-\operatorname{diag}\left(L_{i} C_{\text {ide }}\right)_{i \in V}\right)\right) \xi .
$$

Therefore, we can obtain Theorem 1 as a stability condition of the estimation error of the observer (10).

Both the alternative input observer (10) and the unknown input observer (9) are also solutions to Problem 2. However, the unknown input observer cannot provide the estimate in real time for a network with a communication delay. Therefore, we propose a distributed observer with delay compensation by using the alternative inputs in the next subsection.

\subsection{Alternative Input Observer with Delay Compensation}

An observer with sensor delays was proposed by Watanabe [12]. A delayed output $C_{i} x\left(t-d_{(i, j)}\right)$ can be compensated by adding a delay compensation term $\Xi(t)$, which can be expressed by

$$
\begin{aligned}
& C_{i} x\left(t-d_{(i, j)}\right)+\Xi(t)=C_{i} e^{-A d_{(i, j)}} x(t), \\
& \Xi(t)=C_{i} e^{-A d_{(i, j)}} \int_{t-d_{(i, j)}}^{t} e^{A(t-\tau)} \sum_{i=1}^{N} B_{i} u_{i}(\tau) d \tau .
\end{aligned}
$$

Each node obtains all observed data of the other nodes via the aggregated data (6a) with multiple delays that depend on the communication paths. Therefore, a distributed calculation of the delay compensation terms is necessary. In addition, the unknown input is needed to execute the calculation of the delay compensation in each node. In this paper, we utilize the alternative input in calculating the delay compensation. The delay compensation term sent from node $i$ to $j$ can be expressed by

$$
\begin{aligned}
\bar{\Xi}_{i j}(t) & =\sum_{k \in J_{i} \backslash\{j\}}\left(\bar{\Xi}_{k i}\left(t-d_{(k, i)}\right)\right. \\
& \left.+\bar{C}_{(k, i)} e^{-A d_{(k, i)}} \int_{t-d_{(k, i)}}^{t} e^{A(t-\tau)} \sum_{l=1}^{N} B_{l} \hat{u}_{i l}(\tau) d \tau\right),
\end{aligned}
$$

where $\bar{C}_{(i, j)}$ is a matrix defined by

$$
\bar{C}_{(i, j)}=C_{i}+\sum_{k \in J_{i} \backslash\{j\}} \bar{C}_{(k, i)} e^{-A d_{(k, i)}} .
$$


Note that $\hat{u}_{i i}=u_{i}$ and the calculation of (13) can be executed off-line. Each node calculates $\hat{\Xi}_{i}$ as

$$
\begin{aligned}
\hat{\Xi}_{i}(t) & =\sum_{j \in J_{i}}\left(\bar{\Xi}_{j i}\left(t-d_{(j, i)}\right)\right. \\
& \left.+\bar{C}_{(j, i)} e^{-A d_{(j, i)}} \int_{t-d_{(j, i)}}^{t} e^{A(t-\tau)} \sum_{k=1}^{N} B_{i} \hat{u}_{i k}(\tau) d \tau\right) .
\end{aligned}
$$

By using $\hat{y}_{i}, \hat{\Xi}_{i}$ and the alternative inputs, the observer of node $i$ can be expressed by

$$
\begin{aligned}
\dot{\hat{x}}_{i}(t) & =A \hat{x}_{i}(t)+\sum_{j=1}^{N} B_{j} \hat{u}_{i j}(t) \\
& +L_{i}\left(\hat{y}_{i}(t)+\hat{\Xi}_{i}(t)-\hat{C}_{i} \hat{x}_{i}(t)\right),
\end{aligned}
$$

where $\hat{C}_{i}$ is a matrix given by

$$
\hat{C}_{i}=C_{i}+\sum_{j \in J_{i}} \bar{C}_{(j, i)} e^{-A d_{(j, i)}} .
$$

In the case where each node can obtain all inputs in real time, the estimation error of the observer (15) is asymptotically stable if and only if $A-L_{i} \hat{C}_{i}$ is Hurwitz. On the other hand, the stability condition of the estimation error for (15) under the existence of alternative inputs is given by Theorem 2.

Let $\bar{E}(i)$ be a set of edges whose elements are a part of simple paths from node $j$ to $i$ for all $j \neq i$. For example, $\bar{E}(1)$ in Fig. 3 is given by

$$
\begin{aligned}
\bar{E}(1)= & \{(2,1),(3,1),(4,2),(5,2),(6,3), \\
& (7,3),(8,4),(9,4)\} .
\end{aligned}
$$

The density of $\bar{E}(i)$ is $N-1$ regardless of $i$. For each $i$, we define $P_{i} \in \mathbb{S}_{++}^{n}$ and $M_{i} \in \mathbb{R}^{n \times p}=P_{i} L_{i}$. In addition, for each $i$ and $e \in \bar{E}(i)$, the matrices ${ }^{i} Q_{e} \in \mathbb{S}_{++}^{n N},{ }^{i} W_{e} \in \mathbb{S}_{++}^{n N}$ and ${ }^{i} R_{e} \in \mathbb{S}_{++}^{n}$ are defined. By using ${ }^{i} R_{e},{ }^{i} \Omega_{e}$ is given by

$$
{ }^{i} \Omega_{e=(j, k)}=\int_{0}^{d_{e}} e^{A \theta} \bar{B}_{j}^{i} R_{e}^{-1} \bar{B}_{j}^{T} e^{A^{T} \theta} d \theta .
$$

From $\bar{A}, P_{i}, M_{i}, \hat{C}_{i}$, and ${ }^{i} Q_{e}$, the matrix $\Phi$ is calculated by

$$
\begin{aligned}
\Phi & =\operatorname{He}\left(\operatorname{diag}\left(P_{i}\right)_{i \in V} \bar{A}-\operatorname{diag}\left(M_{i} \hat{C}_{i}\right)_{i \in V}\right) \\
& +\sum_{i=1}^{N} \sum_{e \in \bar{E}(i)}{ }^{i} Q_{e} .
\end{aligned}
$$

We set $\tilde{C}_{i},{ }^{i} \Omega$ as

$$
\tilde{C}_{i}=\operatorname{row}\left(\bar{C}_{e}\right)_{e \in \bar{E}(i)},{ }^{i} \Omega=\operatorname{diag}\left({ }^{i} \Omega_{e}\right)_{e \in \bar{E}(i)} .
$$

Lemma 1. Let us consider the function $V_{1}$ as

$$
V_{1}=\xi^{T}(t) \operatorname{diag}\left(P_{i}\right)_{i \in V} \xi(t) .
$$

The time differentiation of $V_{1}$ satisfies the following inequality:

$$
\begin{aligned}
\dot{V}_{1} & \leq \xi^{T}(t) \Lambda \xi(t) \\
& +\sum_{i=1}^{N} \sum_{e=(k, j) \in \bar{E}(i)} \xi^{T}(t) P_{i} L_{i} \bar{C}_{e}{ }^{i} \Omega_{e} \bar{C}_{e}^{T} L_{i}^{T} P_{i}^{T} \xi(t) \\
& +\sum_{i=1}^{N} \sum_{e=(k, j) \in \bar{E}(i)} \int_{t-d_{(k, i)}}^{t-d_{(j, i)}} \xi^{T}(\tau) \bar{B}_{j}^{T i} R_{e} \bar{B}_{j} \xi(\tau) d \tau, \\
\Lambda & =\operatorname{He}\left(\operatorname{diag}\left(P_{i}\right)_{i \in V}\left(\bar{A}-\operatorname{diag}\left(L_{i} \hat{C}_{i}\right)_{i \in V}\right)\right) .
\end{aligned}
$$

Proof. Let ${ }^{i} \hat{\Xi}_{j k}$ be a delay-compensated term sent from node $j$ to $k$ when node $i$ is a root node. The term ${ }^{i} \hat{\Xi}_{j k}$ can be expressed by

$$
\begin{aligned}
{ }^{i} \hat{\Xi}_{j k}(t) & =\sum_{l \in P_{i j}}\left({ }^{i} \hat{\Xi}_{l j}\left(t-d_{(l, j)}\right)\right. \\
& \left.+\bar{C}_{(l, j)} e^{-A d_{(l, j)}} \int_{t-d_{(l, j)}}^{t} e^{A(t-\tau)} \sum_{p=1}^{N} B_{p} \hat{u}_{j p}(\tau) d \tau\right) .
\end{aligned}
$$

In the protocol (14), each node does not need to distinguish where the node is a root. For example, ${ }^{i} \hat{\Xi}_{13}, i=3,6,7$ are equal to $\bar{\Xi}_{13}$ in Fig. 3. However, the notation of (14) is not convenient for the proof, so we use Eq. (17) in this subsection. Note that ${ }^{i} \hat{\Xi}_{i i}=\hat{\Xi}_{i}$. Let an error between actual inputs and the alternative input be $\tilde{u}_{i j}=u_{j}-\hat{u}_{i j}$. By substituting $\hat{u}_{i j}=u_{j}-\tilde{u}_{i j}$ into the Eq. (17), we can get

$$
\begin{aligned}
{ }^{i} \hat{\Xi}_{j k}(t) & ={ }^{i} \Xi_{j k}(t)+{ }^{i} \tilde{\Xi}_{j k}(t), \\
{ }^{i} \Xi_{j k}(t) & =\sum_{l \in P_{i j}}\left({ }^{i} \Xi_{l j}\left(t-d_{(l, j)}\right)\right. \\
+ & \left.\bar{C}_{(l, j)} e^{-A d_{l, j}} \int_{t-d_{l(, j)}}^{t} e^{A(t-\tau)} \sum_{p=1}^{N} B_{p} u_{p}(\tau) d \tau\right), \\
{ }^{i} \tilde{\Xi}_{j k}(t) & =\sum_{l \in P_{i j}}\left({ }^{i} \tilde{\Xi}_{l j}\left(t-d_{(l, j)}\right)\right. \\
- & \left.\bar{C}_{(l, j)} e^{-A d_{(l, j)}} \int_{t-d_{l l, j)}}^{t} e^{A(t-\tau)} \sum_{p=1}^{N} B_{p} \tilde{u}_{j p}(\tau) d \tau\right) .
\end{aligned}
$$

The term ${ }^{i} \Xi_{j k}$ is the actual delay compensation term from node $j$ to $k$. On the other hand, ${ }^{i} \tilde{\Xi}_{j k}$ is the error between the actual delay compensation term and that based on the alternative inputs. Therefore, ${ }^{i} \Xi_{i i}$ can be expressed by

$$
{ }^{i} \Xi_{i i}(t)=\sum_{j=1}^{N} C_{i} e^{-A d_{(j, i)}} \int_{t-d_{(j, i)}}^{t} e^{A(t-\tau)} \sum_{k=1}^{N} B_{k} u_{k}(\tau) d \tau .
$$

By adding ${ }^{i} \Xi_{i i}$ to $\hat{y}_{i}$, we can compensate the communication delays as

$$
\hat{y}_{i}(t)+{ }^{i} \Xi_{i i}(t)=\hat{C}_{i} x(t) .
$$

Let $\tilde{\Xi}$ be $\tilde{\Xi}=\left[{ }^{1} \tilde{\Xi}_{11}^{T}, \cdots,{ }^{N} \tilde{\Xi}_{N N}^{T}\right]^{T}$. Note that the following 
equation holds:

$$
\sum_{j=1}^{N} B_{j} \tilde{u}_{i j}=-\bar{B}_{i} \xi .
$$

Thus, the dynamics of estimation error $\xi$ for observer (15) can be expressed by

$$
\begin{aligned}
\dot{\xi}(t)= & \left(\bar{A}-\operatorname{diag}\left(L_{i} \hat{C}_{i}\right)_{i \in V}\right) \xi(t)-\operatorname{diag}\left(L_{i}\right)_{i \in V} \tilde{\Xi}(t), \\
{ }^{i} \tilde{\Xi}_{j k}(t) & =\sum_{l \in P_{i j}}\left(i \tilde{\Xi}_{l j}\left(t-d_{(l, j)}\right)\right. \\
& \left.+\bar{C}_{(l, j)} e^{-A d_{(l, j)}} \int_{t-d_{(l, j)}}^{t} e^{A(t-\tau)} \bar{B}_{j} \xi(\tau) d \tau\right) .
\end{aligned}
$$

The time differentiation of $V_{1}$ can be expressed by

$$
\dot{V}_{1}=\xi(t)^{T} \Lambda \xi(t)-2 \sum_{i=1}^{N} \xi(t)^{T} P_{i} L_{i}{ }_{i} \tilde{\Xi}_{i i}(t)
$$

By substituting (18b) into the second term on the right side of Eq. (19), we can obtain

$$
\begin{aligned}
- & 2 \xi^{T}(t) P_{i} L_{i}{ }^{i} \tilde{\Xi}_{i i}(t) \\
= & -2 \sum_{j \in P_{i i}} \xi^{T}(t) P_{i} L_{i}{ }^{i} \tilde{\Xi}_{j i}\left(t-d_{(j, i)}\right) \\
& -2 \sum_{j \in P_{i i}} \int_{t-d_{j, i}}^{t} \xi^{T}(t) P_{i} L_{i} \bar{C}_{(j, i)} e^{A\left(t-\tau-d_{i j}\right)} \bar{B}_{i} \xi(\tau) d \tau .
\end{aligned}
$$

Let $a$ and $b$ be $a, b \in \mathbb{R}^{n}$, and $R$ be $R \in \mathbb{S}_{++}^{n}$. For $a, b$, and $R$, the following inequality holds:

$$
2 a^{T} b \leq a^{T} R^{-1} a+b^{T} R b .
$$

Therefore, the integral term in (20) can be expressed by

$$
\begin{aligned}
& -2 \int_{t-d_{(j, i)}}^{t} \xi^{T}(t) P_{i} L_{i} \bar{C}_{(j, i)} e^{A\left(t-\tau-d_{(j, i)}\right)} \bar{B}_{i} \xi(\tau) d \tau \\
& \leq 2 \int_{t-d_{(j, i)}}^{t}\left|\xi^{T}(t) P_{i} L_{i} \bar{C}_{(j, i)} e^{A\left(t-\tau-d_{(j, i)}\right)} \bar{B}_{i} \xi(\tau)\right| d \tau \\
& \leq \xi^{T}(t) P_{i} L_{i} \bar{C}_{(j, i)}{ }^{i} \Omega_{(j, i)} \bar{C}_{(j, i)}^{T} L_{i}^{T} P_{i}^{T} \xi(t) \\
& +\int_{t-d_{(j, i)}}^{t} \xi^{T}(\tau) \bar{B}_{i}^{T i} R_{(j, i)} \bar{B}_{i} \xi(\tau) d \tau .
\end{aligned}
$$

The first term in (20) can be expressed by

$$
\begin{aligned}
& -2 \xi^{T}(t) P_{i} L_{i}{ }^{i} \tilde{\Xi}_{j i}\left(t-d_{(j, i)}\right) \\
= & -2 \sum_{k \in P_{i j}} \xi^{T}(t) P_{i} L_{i}{ }_{i} \tilde{\Xi}_{k j}\left(t-d_{(k, i)}\right) \\
& -2 \sum_{k \in P_{i j}} \int_{t-d_{(k, i)}}^{t-d_{(j, i)}} \xi^{T}(t) P_{i} L_{i} \bar{C}_{(k, j)} e^{A\left(t-\tau-d_{(k, i)}\right)} \bar{B}_{j} \xi(\tau) d \tau .
\end{aligned}
$$

The integral term in (21) is also expressed by

$$
\begin{aligned}
& -2 \int_{t-d_{(k, i)}}^{t-d_{(j, i)}} \xi^{T}(t) P_{i} L_{i} \bar{C}_{(k, j)} e^{A\left(t-\tau-d_{(k, i)}\right)} \bar{B}_{j} \xi(\tau) d \tau \\
& \leq \xi^{T}(t) P_{i} L_{i} \bar{C}_{(k, j)}{ }^{i} \Omega_{(k, j)} \bar{C}_{(k, j)}^{T} L_{i}^{T} P_{i}^{T} \xi(t) \\
& +\int_{t-d_{(k, i)}}^{t-d_{(j, i)}} \xi^{T}(\tau) \bar{B}_{j}^{T i} R_{(k, j)} \bar{B}_{j} \xi(\tau) d \tau .
\end{aligned}
$$

If node $j$ is the leaf node, ${ }^{i} \tilde{\Xi}_{k j}(t)$ satisfies that ${ }^{i} \tilde{\Xi}_{k j}(t)=0$. Thus, $\dot{V}_{1}$ is recursively given by

$$
\begin{aligned}
\dot{V}_{1} \leq \xi^{T}(t) \Lambda \xi(t) & \\
& +\sum_{i=1}^{N} \sum_{e=(k, j) \in \bar{E}(i)} \xi^{T}(t) P_{i} L_{i} \bar{C}_{e}{ }^{i} \Omega_{e} \bar{C}_{e}^{T} L_{i}^{T} P_{i}^{T} \xi(t) \\
& +\sum_{i=1}^{N} \sum_{e=(k, j) \in \bar{E}(i)} \int_{t-d_{(k, i)}}^{t-d_{(j, i)}} \xi^{T}(\tau) \bar{B}_{j}^{T i} R_{e} \bar{B}_{j} \xi(\tau) d \tau .
\end{aligned}
$$

Theorem 2. If there exist matrices $P_{i}, M_{i},{ }^{i} Q_{e},{ }^{i} W_{e}$, and ${ }^{i} R_{e}$ that satisfy

$$
\begin{aligned}
& \left(\begin{array}{cc}
\operatorname{diag}\left({ }^{i} \Omega^{-1}\right)_{i \in V} & \operatorname{diag}\left(\tilde{C}_{i}^{T} M_{i}^{T}\right)_{i \in V} \\
\operatorname{diag}\left(M_{i} \tilde{C}_{i}\right)_{i \in V} & -\Psi
\end{array}\right)>0 \\
& d_{e}{ }^{i} W_{e}-{ }^{i} Q_{e}<0 \\
& \bar{B}_{j}^{T i} R_{e} \bar{B}_{j}-{ }^{i} W_{e}<0
\end{aligned}
$$

then the estimation error $\xi_{i}=x-\hat{x}_{i}$ of (15) is stable.

Proof. We consider $V=V_{1}+V_{2}+V_{3}$ as a Lyapunov Krasovskii functional candidate, where $V_{2}$ and $V_{3}$ are expressed by

$$
\begin{aligned}
& V_{2}=\sum_{i=1}^{N} \sum_{e=(k, j) \in \bar{E}(i)} \int_{t-d_{(k, i)}+d_{e}}^{t} \xi^{T}(\tau)^{i} Q_{e} \xi(\tau) d \tau, \\
& V_{3}=\sum_{i=1}^{N} \sum_{e=(k, j) \in \bar{E}(i)} \int_{-d_{e}}^{0} \int_{t-d_{(k, i)}+d_{e}+\theta}^{t-d_{(k, i)}+d_{e}}{ }^{i} V_{e}(\tau) d \tau d \theta \\
& { }^{i} V_{e}(\tau)=\xi^{T}(\tau)^{i} W_{e} \xi(\tau) .
\end{aligned}
$$

The time differentiations of $V_{2}$ and $V_{3}$ are given by

$$
\begin{aligned}
& \dot{V}_{2}=\sum_{i=1}^{N} \sum_{e=(k, j) \in \bar{E}(i)}\left(\xi^{T}(t)^{i} Q_{e} \xi(t)\right.\left.-\xi^{T}\left(t-d_{(j, i)}\right)^{i} Q_{e} \xi\left(t-d_{(j, i)}\right)\right) \\
& \dot{V}_{3}=\sum_{i=1}^{N} \sum_{e=(k, j) \in \bar{E}(i)}\left(d_{e} \xi^{T}\left(t-d_{(j, i)}\right)^{i} W_{e} \xi\left(t-d_{(j, i)}\right)\right. \\
&\left.-\int_{t-d_{(k, i)}}^{t-d_{(j, i)}} \xi^{T}(\tau)^{i} W_{e} \xi(\tau) d \tau\right) .
\end{aligned}
$$


From Lemma 1 and Eq. (25), we can obtain the inequality about $\dot{V}$ as

$$
\begin{aligned}
\dot{V} & \leq \xi^{T}(t) \Psi \xi(t) \\
& +\sum_{i=1}^{N} \sum_{e=(k, j) \in \bar{E}(i)} \xi^{T}\left(t-d_{(j, i)}\right)\left(d_{e}{ }^{i} W_{e}-{ }^{i} Q_{e}\right) \xi\left(t-d_{(j, i)}\right) \\
& +\sum_{i=1}^{N} \sum_{e=(k, j) \in \bar{E}(i)} \int_{t-d_{(k, i)}}^{t-d_{(j, i)}} \xi^{T}(\tau)\left(\bar{B}_{j}^{T i} R_{e} \bar{B}_{j}-{ }^{i} W_{e}\right) \xi(\tau) d \tau,
\end{aligned}
$$

where $\Psi$ is given by

$$
\Psi=\Phi+\sum_{i=1}^{N} \sum_{e=(k, j) \in \bar{E}(i)} P_{i} L_{i} \bar{C}_{e}{ }^{i} \Omega_{e} \bar{C}_{e}^{T} L_{i}^{T} P_{i}^{T} .
$$

The time differentiation of $V$ satisfies $\dot{V}<0$ except for $\xi=0$ if (23b), (23c), and the following matrix inequalities hold:

$$
\Psi<0 .
$$

By applying Schur complements to (26), (23a) is obtained.

The Eq. (23) is not the LMI for $P_{i}, M_{i},{ }^{i} Q_{e},{ }^{i} W_{e}$, or ${ }^{i} R_{e}$. However the Eq. (16) can be transformed into

$$
\operatorname{He}\left(A^{i} \Omega_{e}\right)=e^{A d_{e} i} R_{e}^{-1} e^{A^{T} d_{e}}-{ }^{i} R_{e}^{-1} .
$$

Therefore, the Eq. (23) becomes the LMI for $P_{i}, M_{i},{ }^{i} Q_{e}$, ${ }^{i} W_{e},{ }^{i} R_{e}$, and ${ }^{i} \Omega_{e}$ with equality constraints. However, in the numerical calculation of a semidefinite programming problem, the equality constraint is undesirable. Thus, it is convenient that ${ }^{i} R_{e}$ is given in the actual calculation.

To solve (23), all delay values are already known and invariant. This assumption is not practical because there are communication jitters owing to modeling errors/disturbances in actual wireless networks. However, the LMI can be utilized to design the controller for a system with uncertainty. Therefore, our proposed method can be applied to a system with unknown communication delays with some modifications.

From Eq. (11), we can confirm that if we can select the observer gains of each node to be sufficiently high, then the estimation error of each node converges to 0 in the no-delay case. This result does not contradict the motivation of the introduction to the alternative inputs. If a communication delay exists, then the stability condition (23a) can be transformed into Eq. (26). From Eq. (26), it is also necessary that the observer gains of each node are high to estimate the state. On the other hand, it is also confirmed that when we raise the observer gain too high, Eq. (26) is not satisfied. Thus, there exist some systems that have no solution to (23). For example, let us consider systems whose states are given by a scalar. In this case, (26) becomes a quadratic inequality against the observer gains. Hence, the observer gains are bounded to satisfy (26). In addition, there exists no solution to (26) if ${ }^{i} Q_{e}$ becomes large. Therefore, it is difficult for us to obtain the observer for a system with high-gain controllers or large delay networks because (23b) and (23c) indicate that these systems require a large ${ }^{i} Q_{e}$.

\section{Numerical Simulation}

In this section, we consider a linear system with 10 nodes that can be expressed by

$$
\left(\begin{array}{c}
\dot{x}_{1} \\
\dot{x}_{2}
\end{array}\right)=\left(\begin{array}{cc}
0.050 & -0.105 \\
0.015 & 0.085
\end{array}\right)\left(\begin{array}{l}
x_{1} \\
x_{2}
\end{array}\right)+\sum_{i=1}^{10} B_{i} u_{i}
$$

The network that observes and controls system (27) has a topology shown by Fig. 4 . The odd-numbered nodes can measure $x_{1}$ and inject the input to $x_{2}$. On the other hand, the even-numbered nodes can measure $x_{2}$ and inject the input to $x_{1}$. Therefore, the input and output matrices of each node are given by

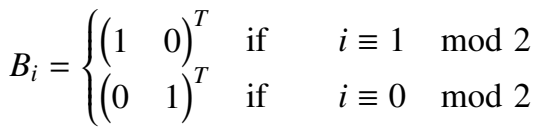

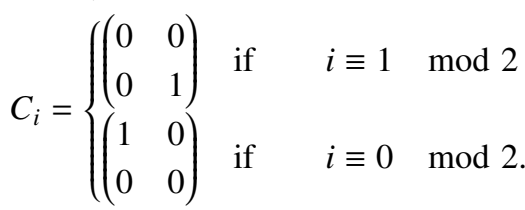

The communication delays in the network are uniformly 0.25 . The feedback gains of each node $K_{i}$ are determined such that the eigenvalues of the following matrix (28) become $(-0.05,-0.1)$.

$$
A+\sum_{i=1}^{10} B_{i} K_{i}
$$

We give the parameter matrices ${ }^{i} R_{e}$ as, uniformly, $I_{2} \times 0.001$. Under the above conditions, we solve the LMI (23) to find the observer gains of each node. We can confirm that some observer gains become height compared to the system matrix of (27). For example, we can obtain the observer gain for the 2 nd node as

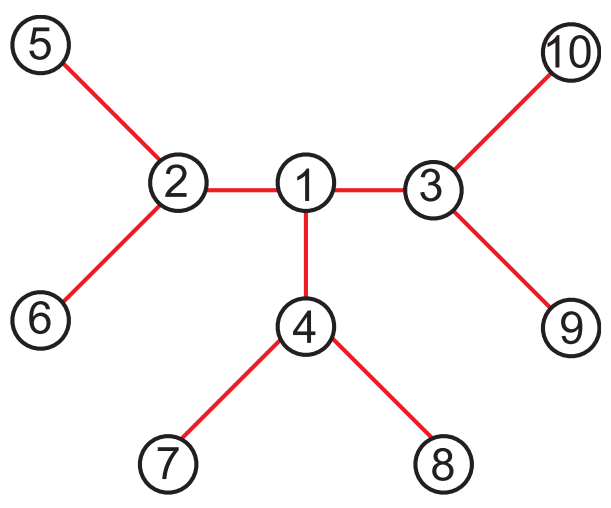

Fig. 4 Topology of network. 


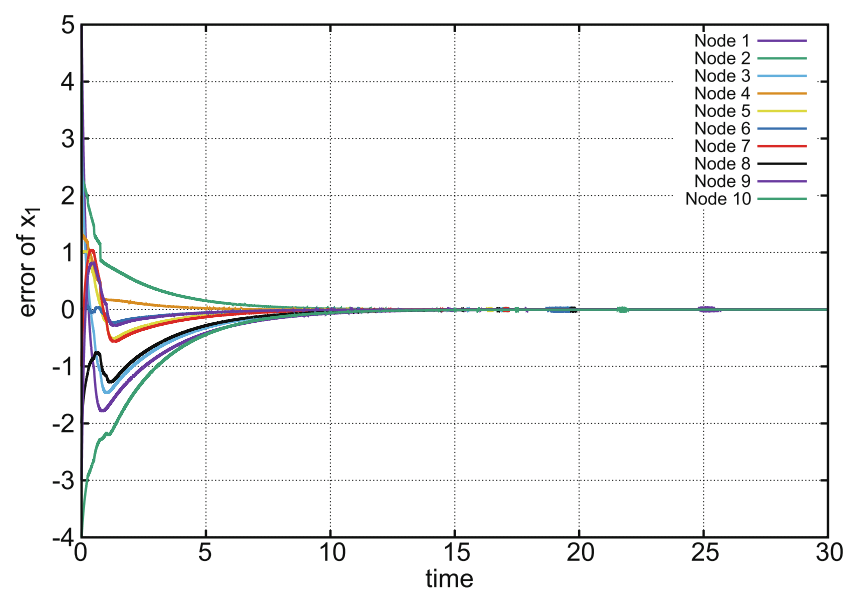

Fig. 5 Time response of estimation error on $x_{1}$.

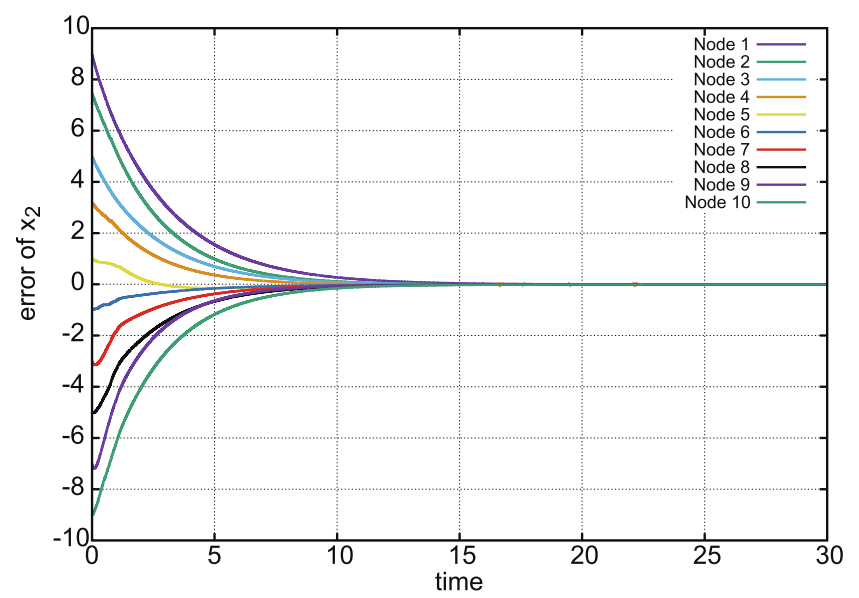

Fig. 6 Time response of estimation error on $x_{2}$.

$$
L_{2}=\left(\begin{array}{cc}
36.9160 & -10.9542 \\
-10.0549 & 2.9931
\end{array}\right)
$$

The time responses of the estimation errors on $x_{1}$ and $x_{2}$ are shown in Figs. 5 and 6. It is also confirmed that the estimation errors converge to 0 .

From a series of other numerical simulations, we conclude that the condition (23) is very severe for the system condition. Of course, there exist cases in which the LMI (23) becomes feasible, as in the example above. However, in many cases, for example, a case in which the feedback gain is high, the system matrix $A$ has a large unstable eigenvalue, or the communication delay is large, the LMI (23) becomes infeasible. On the other hand, if the communication delay is small enough to be ignored, then we can estimate the state from the alternative input observer with high gain. In addition, if each node can obtain all inputs of the other nodes, then the observer (15) can estimate the state if and only if $\left(A, \hat{C}_{i}\right)$ is an observable pair. Therefore, it is important for us to collect either the input or the output in real time to estimate the state without delays.

\section{Conclusion}

In this paper, we proposed an alternative input observer for a sensor network with communication delays. Each node estimates the state and calculates the input from its own estimate. To calculate the observer and the delay compensation, each node utilizes the alternative input that is calculated from its own estimate instead of the input of the other nodes. The stability condition of the alternative input observer is given by the LMI.

We introduced a stability condition from the LyapunovKrasovskii functional analysis, which means that the result of this paper is conservative. We may be able to relax the stability condition of the alternative input observer under the existence of a communication delay. However, we conclude that it is important for us to collect either the input or the output in real time to estimate the state without the delays.

This work was partly supported by JSPS KAKENHI Grant Numbers JP17K06486, JP19H02157, and JP19H02158.

\section{References}

[1] R. Olfati-Saber, "Distributed Kalman filtering for sensor networks," Decision and Control, 2007 46th IEEE Conference on, pp.54925498, IEEE, 2007.

[2] R. Olfati-Saber, "Distributed Kalman filter with embedded consensus filters," Decision and Control, 2005 and 2005 European Control Conference. CDC-ECC'05. 44th IEEE Conference on, pp.81798184, IEEE, 2005.

[3] R. Olfati-Saber and J.S. Shamma, "Consensus filters for sensor networks and distributed sensor fusion," Decision and Control, 2005 and 2005 European Control Conference. CDC-ECC'05. 44th IEEE Conference on, pp.6698-6703, IEEE, 2005.

[4] R. Olfati-Saber and R.M. Murray, "Consensus problems in networks of agents with switching topology and time-delays," IEEE Trans. Autom. Control, vol.49, no.9, pp.1520-1533, 2004.

[5] S. Boyd, A. Ghosh, B. Prabhakar, and D. Shah, "Randomized gossip algorithms," IEEE/ACM Trans. Netw. (TON), vol.14, no.SI, pp.2508-2530, 2006.

[6] R. Rajagopalan and P.K. Varshney, "Data aggregation techniques in sensor networks: A survey," IEEE Commun. Surveys Tuts., vol.8, no.4, pp.48-63, 2006.

[7] S. Sundaram and C.N. Hadjicostis, "Delayed observers for linear systems with unknown inputs," IEEE Trans. Autom. Control, vol.52, no.2, pp.334-339, 2007.

[8] W. Chen and M. Saif, "Unknown input observer design for a class of nonlinear systems: An LMI approach," American Control Conference, 2006, pp.5-pp, IEEE, 2006.

[9] M. Darouach, M. Zasadzinski, and S.J. Xu, "Full-order observers for linear systems with unknown inputs," IEEE Trans. Autom. Control, vol.39, no.3, pp.606-609, 1994.

[10] R. Adachi, Y. Yamashita, and K. Kobayashi, "Distributed estimation over delayed sensor network with scalable communication," IEICE Trans. Fundamentals, vol.E102-A, no.5, pp.712-720, May 2019.

[11] D. Tsubakino, M. Krstic, and T.R. Oliveira, "Exact predictor feedbacks for multi-input LTI systems with distinct input delays," Automatica, vol.71, pp.143-150, 2016.

[12] K. Watanabe and M. Ito, "An observer for linear feedback control laws of multivariable systems with multiple delays in controls and outputs," Syst. Control Lett., vol.1, no.1, pp.54-59, 1981.

[13] E. Fridman, "New Lyapunov-Krasovskii functionals for stability of 
linear retarded and neutral type systems," Syst. Control Lett., vol.43, no.4, pp.309-319, 2001.
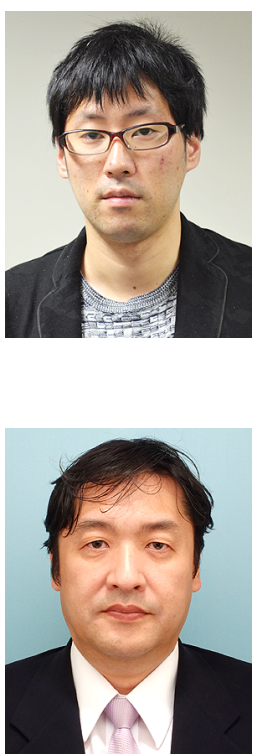

of the SICE, ISCIE, RSJ, and IEEE.

Ryosuke Adachi received his B.E., M.I.S. and $\mathrm{Ph} . \mathrm{D}$. degrees from Hokkaido University, Japan, in 2014, 2016 and 2019, respectively. Since 2019, he has been an Assistant Professor at the Graduate School of Sciences and Technology for Innovation at Yamaguchi University. His research interests include analysis and control of networked systems. He is a member of the SICE, ISCIE, and IEEE.

Yuh Yamashita received his B.S., M.S., and $\mathrm{Ph} . \mathrm{D}$. degrees from Hokkaido University, Japan, in 1984, 1986, and 1993, respectively. In 1988 , he joined the faculty of Hokkaido University. From 1996 to 2004, he was an Associate Professor at the Nara Institute of Science and Technology, Japan. Since 2004, he has been a Professor of the Graduate School of Information Science and Technology, Hokkaido University. His research interests include nonlinear control and nonlinear dynamical systems. He is a member

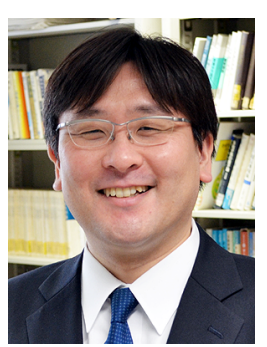

Koichi Kobayashi received the B.E. degree in 1998 and the M.E. degree in 2000 from Hosei University, and the D.E. degree in 2007 from Tokyo Institute of Technology. From 2000 to 2004, he worked at Nippon Steel Corporation. From 2007 to 2015, he was an Assistant Professor at Japan Advanced Institute of Science and Technology. Since 2015, he has been an Associate Professor at the Graduate School of Information Science and Technology, Hokkaido University. His research interests include analysis and control of discrete event and hybrid systems. He is a member of the IEEE, IEEJ, IEICE, and ISCIE. 